Chirurg 2014 · 85:824

DOI 10.1007/s00104-014-2859-y

Online publiziert: 14. August 2014

c) Springer-Verlag Berlin Heidelberg 2014

J. Reibetanz · C.-T. Germer

Klinik für Allgemein-, Viszeral-, Gefäß- und Kinderchirurgie, Universitätsklinikum Würzburg

\title{
Anteriores Resektionssyndrom und Lebensqualität bei Rektumkarzinompatienten
}

Juul T, Ahlberg M, Biondo S et al (2014) Low anterior resection syndrome and quality of life: an international multicenter study. Dis Colon Rectum 57(5):585-591

\section{Hintergrund und Fragestellung}

Bis zu $50-90 \%$ der Patienten nach tiefer anteriorer Rektumresektion (TAR) leiden postoperativ unter dem sog. anterioren Resektionssyndrom („low anterior resection syndrom", LARS), charakteristiert durch u. a. Inkontinenz, „urgency“, fraktionierte sowie häufige Stuhlentleerungen. Ziel dieser Studie war die Untersuchung des Zusammenhangs zwischen dem Auftreten eines LARS und der postoperativen Lebensqualität (QoL) bei Rektumkarzinompatienten unter Verwendung validierter Fragebögen.

\section{Methode}

Fünf Zentren aus vier europäischen Ländern waren an der Studie beteiligt. Eingeschlossen waren Patienten nach kurativer Resektion eines Rektumkarzinoms, wobei die Operation mindestens 16 Monate zurückliegen musste, um von einer stabilen postoperativen Darm-/Entleerungsfunktion ausgehen zu können. Die Datenerhebung erfolgte mittels validierter Fragebögen:

- dem EORTC QOL-C30, einem Fragebogen zur allgemeinen Lebensqualität von Karzinompatienten, sowie

- dem LARS-Score, einem aus 5 Einzelfragen bestehenden Fragebogen zur
Analyse der funktionellen Beschwerden bei Patienten nach TAR.

\section{Diskussion und Fazit des Reviewers}

Letzterer teilt die Beschwerden anhand eines Punktwertes (0-42 Punkte) grob in drei Schweregrade ein: kein LARS, minorLARS und major-LARS.

\section{Ergebnisse}

Von insgesamt 1061 eingeschlossenen $\mathrm{Pa}$ tienten standen die Daten von 796 Patienten zur Verfügung, was einer Respondsrate von $75 \%$ entsprach. $56 \%$ der befragten Patienten litten unter einem stark ausgeprägtem anteriorem Resektionssyndrom (major-LARS), 19\% der Patienten gaben milde Beschwerden an (minorLARS), nur 29\% der Patienten hatten keine Zeichen eines anterioren Resektionssyndroms. Verglichen mit Patienten ohne anteriores Resektionssyndrom zeigten Patienten mit major-LARS eine deutliche Einschränkung ihrer allgemeinen postoperativen Lebensqualität. Hier fanden sich klinisch relevante (Punktabweichung $\geq 10)$ und statistisch signifikante $(\mathrm{p}<0,01)$ Unterschiede in 7 von 8 ausgewählten Subskalen des EORTC-QLQ-C30-Fragebogens. Patienten mit mildem LARS hatten zwar in einigen Subskalen des QoLFragebogens eine (statistisch-signifikant) schlechtere QoL als Patienten ohne LARS, die Unterschiede waren aber insgesamt zu gering, um klinisch relevant zu sein.
In den vergangenen Jahren konnte durch frühere Diagnostik und multimodale Therapiekonzepte die Prognose von Patienten mit Rektumkarzinom deutlich verbessert werden. Folglich treten neben den onkologischen Ergebnissen auch zunehmend Aspekte der postoperativen Lebensqualität in den Vordergrund. Die aktuelle Arbeit leistet hierzu einen wichtigen Beitrag und zeigt eindrücklich, dass die Lebensqualität von Patienten nach TAR sehr stark vom Auftreten und dem Schweregrad eines anterioren Resektionssyndroms abhängig ist. Da hiervon bis zu zwei Drittel aller Patienten nach TAR betroffen sein können, sollten unsere therapeutischen Bemühungen (auch weiterhin) nicht nur auf onkologische, sondern auch auf funktionelle Ergebnisse der Operation ausgerichtet sein.

\section{Korrespondenzadresse}

Prof. Dr. C.-T. Germer

Klinik für Allgemein-, Viszeral-, Gefäß- und Kinderchirurgie, Universitätsklinikum Würzburg, Oberdürrbacher Str. 6, 97080 Würzburg germer_c@ukw.de

Interessenkonflikt. J. Reibetanz und C.-T. Germer geben an, dass kein Interessenkonflikt besteht. 